\title{
EL TURISMO DE NIEVE COMO ESTRATEGIA DE DESARROLLO EN EL PIRINEO ARAGONÉS
}

\author{
T. LASANTA \\ Instituto Pirenaico de Ecología (CSIC). \\ Campus de Aula Dei. Apdo. 13034 - Zaragoza \\ Correo electrónico de contacto: fm@ipe.csis.es
}

\begin{abstract}
RESUMEN. Entre 1965 y 1976 se construyeron cinco estaciones de esquí alpino en el Pirineo aragonés (Candanchú, con antecedentes desde 1920, Formigal, Cerler, Panticosa y Astún), con el fin de potenciar las actividades turísticas y romper con una situación socioeconómica regresiva, por la emigración de la población y la crisis del sector primario, iniciada a principios del siglo XX. En este trabajo se analiza: i) la influencia que las estaciones de esquí han tenido en la evolución de la población, en la estructura demográfica y en el empleo, y ii) la incidencia del desarrollo del sector turístico en las explotaciones primarias y en los censos ganaderos. Los resultados obtenidos permiten concluir que en los municipios afectados por las estaciones de esquí la población aumentó el 60,4\% entre 1970 y 2008, contando con una estructura demográfica relativamente equilibrada y una concentración del empleo en el sector servicios (82,2\%) y en la construcción $(13,1 \%)$, mientras que en el sector primario sólo trabajan el 2,6\% de los activos. Los municipios sin estaciones de esquí pierden el 26,6\% de su población entre 1970 y 2008, mostrando una estructura envejecida y una alta tasa de dependencia (55,7\%), si bien mantienen una distribución del empleo más equilibrada, con el 19,5\% de los trabajadores en el sector primario. La evolución del número de explotaciones agropecuarias pone de manifiesto la mayor caída en los municipios con estaciones (48\% entre 1970 y 2008) que sin estaciones (22\%, entre ambas fechas). Parecido comportamiento se observa en la evolución de los censos ganaderos, donde los municipios con estaciones perdieron el $43 \%$, mientras que los municipios sin estaciones incrementaron su censo en el $31,8 \%$ en el mismo período.
\end{abstract}

ABSTRACT. Five alpine ski resorts were built in the Pyrenees between 1965 and 1976: Candanchú (with a previous history dating back to 1920), Formigal, Cerler, Panticosa and Astún. The aim was to develop tourist activities and improve a regressive socio-economical situation, in which the population abandoned progressively the area that had suffered a severe crisis in the Primary Productive Sector since the beginning of the XXth Century. In this paper two factors have been 
analyzed: i) the influence that ski resorts have had in the evolution of the human population, in demographic structure and in employment, and ii) the impact of the tourist sector on the Primary Productivity farms, and on the livestock census. The results obtained permit the conclusion that in the municipalities affected by the ski resorts, the population has grown $60.4 \%$ between 1970 and 2008, showing a fairly balanced demographic structure and a concentration of employment in the sectors of Services (82.26\%) and building construction (13.1\%) whereas the primary sector only occupies $2.6 \%$ of active population. The municipalities without ski resorts have lost $26.6 \%$ of their population between 1970 and 2008, show an aged demographic structure and a high dependency level rate (55.7\%) although the employment distribution is more balanced, with $19.5 \%$ of workers in the primary sector. The evolution of the number of farms shows a greater decline in the municipalities with ski resorts (48\% between 1970 and 2008), than in those without ski resorts (22\% during the same period of time). Similar behaviour can be observed in the evolution of livestock census, where the municipalities with ski resorts have lost $43 \%$ of their livestock while, on the contrary, the municipalities without ski resorts increased their census by $31.8 \%$ during the same period.

Palabras clave: Estaciones de esquí, turismo de montaña, montañas mediterráneas, Pirineos.

Key words: Ski reports, mountain tourist, Mediterranean mountains, Pyrenees.

Enviado el 12 de noviembre de 2009 Aceptado el 7 de abril de 2010

\section{Introducción}

Por encima del piso forestal las montañas cuentan con dos recursos estacionales: la nieve y el pasto. La presencia de la nieve puede llegar hasta los siete meses (de noviembre a mayo) en las montañas españolas de ambiente continental como el Pirineo (García-Ruiz et al., 1985 y 1986), mientras que su permanencia se reduce considerablemente en las de condiciones oceánicas (Cantábrica) o localizadas en latitudes más bajas (Sistema Ibérico, Cordillera Central, Béticas) (López-Moreno et al., 2007).

Hasta muy recientemente la nieve servía casi exclusivamente para mantener estable la temperatura del suelo y favorecer el desarrollo de los pastos alpinos y subalpinos. Por el contrario, los pastos constituyeron un recurso fundamental para las economías de montaña. En torno a ellos surgieron potentes explotaciones de ovino que trashumaban a tierra llana durante la estación fría, una vez que la nieve hacía nuevamente acto de presencia en la montaña (Balcells, 1976).

A lo largo del siglo $\mathrm{XX}$, sin embargo, la nieve va adquiriendo mayor importancia socioeconómica. Se utiliza para la práctica del esquí (Arnáez, 1981; Callizo, 1991) y como reserva de agua, que es acumulada en los embalses para abastecimiento de ciudades, polí- 
gonos industriales y campos de regadío (López-Moreno et al., 2008). El turismo ligado a la nieve constituye recientemente un sector económico fundamental en algunos municipios de la montaña española. Lo cierto es que desde los años sesenta se han construido 29 estaciones de esquí alpino, de las que 16 se localizan en el Pirineo (Candanchú, Astún, Formigal, Panticosa-Los Lagos, Cerler, Baqueíra Beret, Vallter 2000, Vall de Nuria, Masella, Tavascan, Espot E., Boí, Rasos de Peguera, Port Aine, La Molina y Port del Conte), 5 en la Cantábrica (Leitariegos, Valgrande, San Isidro, Alto Campoo y La Manzaneda), 3 en el Sistema Ibérico (Valdezcaray, Valdelinares y Javalambre), 4 en el Sistema Central (La Covatilla, Navacerrada, Valdesquí y La Pinilla) y 1 en las Béticas (Sierra Nevada), a lo que hay que unir las estaciones de esquí de fondo y otras actividades deportivas ligadas a la nieve.

El aprovechamiento económico de la nieve sigue en España planteamientos ya ensayados en otras montañas europeas (Zimmermann, 1994; Ferrero, 1998; Godde et al., 2000), buscando una fuente complementaria de rentas para paliar las pérdidas generadas por la caída de los ingresos de las actividades tradicionales. Se trata, en el fondo, de encontrar nuevas alternativas económicas que permitan reequilibrar el territorio y evitar el abandono del sector primario y la sangría demográfica que la montaña española sufre desde mediados del siglo XX.

Interesa ahora, cuando ya existe cierta perspectiva temporal, averiguar hasta qué punto la creación de estaciones de esquí ha servido como motor económico para reactivar una zona que se venía hundiendo demográficamente y que venía perdiendo en las últimas décadas buena parte de su capacidad productiva. Interesa también comprobar en qué medida los efectos de las estaciones de esquí pueden considerarse como plenamente positivos para lo que debe ser un desarrollo general armónico del territorio y, por último, analizar si existe una integración -que sería muy deseable- entre las nuevas actividades y las tradicionales. Desde esta perspectiva, y centrándonos en el Pirineo aragonés, los objetivos que persigue este trabajo son: i) Conocer la influencia que las estaciones de esquí alpino han tenido en la evolución y estructura demográfica, así como en el empleo y ii) Estudiar la incidencia del desarrollo del sector turístico en la integracióncompetencia con las actividades primarias.

\section{2. Área de estudio}

El área de estudio corresponde al sector más septentrional del Pirineo Aragonés. Constituye una unidad física y de gestión bastante homogénea. Se trata de valles paralelos con una orientación N-S, transversal a la disposición del relieve, que sigue una orientación NW-SE. El relieve es vigoroso, con dominio de las laderas sobre los espacios llanos, que prácticamente quedan limitados a los fondos de valle y a pequeños rellanos a media ladera, con frecuencia de origen glaciolacustre (García-Ruiz y Sala, 1984). Una proporción importante del área de estudio supera los $2.000 \mathrm{~m}$ de altitud. La isoterma anual del $0^{\circ} \mathrm{C}$ se alcanza a los $2.726 \mathrm{~m}$ (Del Barrio et al., 1990), descendiendo en la estación fría hasta los 1.700 m (García-Ruiz et al., 1985). Estas circunstancias hacen que la precipitación en forma de nieve sea frecuente y que el manto nivoso permanezca durante 7-8 meses al año (López-Moreno y García-Ruiz, 2004). 
El modelo tradicional de gestión del territorio se basó en la utilización de las laderas bajas con agricultura cerealista para alimentación de las personas y en el aprovechamiento de los pastos, sobre todo de los alpinos y subalpinos, con ovino trashumante (García-Ruiz y Lasanta, 1990). Ambas actividades iniciaron su decadencia a finales del siglo XIX, de forma que a mediados del siglo XX, el 75\% del espacio agrícola se había abandonado y los censos ganaderos se habían reducido drásticamente (García-Ruiz y Balcells, 1978). La crisis profunda de la agricultura y ganadería coincidió en el tiempo con un intenso proceso de industrialización en áreas urbanas, lo que incentivó la emigración de la población rural, especialmente en la montaña, a las ciudades (Caravaca y Méndez, 1994). Los valles aragoneses, en concreto, perdieron el 38\% de la población entre 1950 y 1970 , pasando de 24.735 a 15.392 habitantes.

En este contexto de decadencia se consideró que la instalación de estaciones de esquí alpino era una alternativa para detener el retroceso socioeconómico e impulsar el desarrollo y reequilibrio territorial del Pirineo. Al amparo de la Ley 197/1963 de 28 de Diciembre sobre Centros y Zonas de Interés Turístico Nacional se levantaron las 5 estaciones de esquí alpino que en la actualidad existen en el Pirineo Aragonés, cuyos principales rasgos se anotan en la Tabla 1. Candanchú fue la primera estación que se montó, aprovechando unas modestas instalaciones previas (Domingo, 2000, apunta que en las laderas de Candachú ya se practicaba el esquí en 1920), mientras que la próxima de Astún fue la última. En conjunto reúnen $277 \mathrm{Km}$ esquiables (la tercera parte de la superficie de pistas balizadas en las estaciones españolas), que se distribuyen por 261 pistas. Admiten 110.537 esquiadores hora y ofertan en su área de influencia 38.320 camas, de las que el $13 \%$ se localiza en las estaciones.

Tabla 1. Ficha básica de las estaciones de esquí alpino del Pirineo Aragonés.

\begin{tabular}{|l|c|c|c|c|c|}
\hline & Astún & Candanchú & Cerler & Formigal & Panticosa \\
\hline Municipio al que pertenece & Jaca & Aísa & Benasque & Sallent & Panticosa \\
\hline Año de inauguración & 1976 & 1965 & 1971 & 1965 & 1970 \\
\hline Km esquiables & 41 & 59 & 60 & 83 & 34 \\
\hline $\mathrm{N}^{\text {o de pistas }}$ & 48 & 53 & 51 & 71 & 38 \\
\hline $\mathrm{N}^{\text {o de remontes }}$ & 14 & 23 & 29 & 23 & 15 \\
\hline Esquiadores/hora & 16908 & 21300 & 24920 & 34054 & 13355 \\
\hline Cañones de nieve & 120 & 134 & 312 & 282 & 100 \\
\hline Cota máxima (m) & 2300 & 2400 & 2630 & 2258 & 2200 \\
\hline Cota mínima (m) & 1700 & 1530 & 1740 & 1740 & 1500 \\
\hline Desnivel & 600 & 870 & 890 & 518 & 700 \\
\hline Alojamiento estación (n ${ }^{\circ}$ camas) & 520 & 1174 & 879 & 1688 & 676 \\
\hline Alojamiento alrededores (n ${ }^{\circ}$ camas) & 10250 & 10538 & 4531 & 6354 & 6647 \\
\hline
\end{tabular}




\section{Métodos}

Se consultó la información incluida en estadísticas oficiales (INE, Gobierno de Aragón y Diputación de Huesca) de cada uno de los 36 municipios del área de estudio. En concreto, se anotó la referida al número de habitantes desde 1950 a 2008, la composición de la población en función de la edad y actividad laboral en 2008, el censo de cabezas de ganado en 1970 y 2008, el número de explotaciones primarias en 1970 y 2008, la superficie de suelo construido y de solares en 2008, la fecha de construcción de los edificios, el número de residencias principales y secundarias, y las plazas de alojamiento turístico desde 1950 a 2008.

Para conocer el grado de crecimiento turístico a escala de municipio se tuvo en cuenta el número de plazas turísticas o de alojamiento. Estas se calcularon sumando el número de camas que dan las estadísticas oficiales para los hoteles, apartamentos, albergues y viviendas de turismo rural, 5 por cada segunda residencia y 0,1 por cada plaza de camping.

Se calcularon varios índices demográficos para conocer la estructura poblacional a escala municipal. Entre ellos se incluyen las Tasas de Dependencia (TD) y Feminidad (TF). Para ello se aplicaron las siguientes fórmulas:

$\mathrm{TD}=($ Pob. menor de 15 años + Pob. mayor de 64 años/ Pob. de 15 a 64 años $)$ x 100. $\mathrm{TF}=($ Pob. Total de mujeres/ Pob. Total de hombres $) \times 100$

\section{Resultados}

\subsection{Influencia de las estaciones de esquí en la evolución del alojamiento turístico} (1971-2008)

En el área de estudio se incluyen 36 municipios, que pueden distribuirse en dos grupos en función de su grado de afectación por las estaciones de esquí. Lasanta et al. (2007) comprobaron que las estaciones de esquí afectan a un área espacial bastante reducida, que coincide con los municipios donde se localizan y con los más próximos y accesibles. En concreto, Candanchú y Astún ejercen una alta influencia en los municipios de Aísa, Canfranc, Villanúa y Castiello de Jaca; Formigal y Panticosa en Sallent de Gállego, Panticosa y Biescas, y Cerler en Benasque y Sahún. Todos estos municipios componen el grupo I. El grupo II corresponde a los municipios poco o nada influidos por las estaciones de esquí (Tabla 2).

En 1955 en el área de estudio había 16 hoteles con un total de 638 camas. El 61,6\% del alojamiento turístico se concentraba en Panticosa (6 hoteles y 393 camas), debido a la atracción de su balneario que puede ser considerado como el primer foco turístico del Pirineo aragonés. Broto (con 1 hotel de 24 habitaciones) y Torla (con 1 hotel de 23 habitaciones) mostraban ya tímidamente la atracción que en el futuro ejercerá Ordesa. Por su parte, Canfranc y Sallent de Gállego contabilizaban 5 hoteles y 136 camas (el 21,3\% del total), que se orientaban más a servicios comerciales y de aduana, derivados de ser pue- 
blos frontera, que al turismo. La oferta turística la completaban Benasque (con 2 hoteles que sumaban 8 camas) y Castejón de Sos (1 hotel de 54 camas). En los años sesenta, la oferta de alojamiento turístico se amplió muy poco y tuvo lugar en los mismos municipios que ya contaban con hoteles (Lasanta y Laguna, 2002).

Tabla 2. Distribución de los municipios en función del grado de afectación por las estaciones de esquí.

\begin{tabular}{|l|l|}
\hline \multicolumn{1}{|c|}{ GRUPO I } & \multicolumn{1}{c|}{ GRUPO II } \\
\hline $\begin{array}{l}\text { Aísa, Benasque, Biescas, Canfranc, Castie- } \\
\text { llo de Jaca, Panticosa, Sahún, Sallent de } \\
\text { Gállego y Villanúa. }\end{array}$ & $\begin{array}{l}\text { Ansó, Aragüés, Bielsa, Bisaurri, Bonansa, } \\
\text { Borau, Broto, Castejón de Sos, Chía, Fago, }\end{array}$ \\
& $\begin{array}{l}\text { Fanlo, Gistaín, Hoz de Jaca, Jasa, Laspaú- } \\
\text { les, Laspuña, Montanuy, Plan, Puértolas, }\end{array}$ \\
& San Juan de Plan, Seira, Sesué, Tella-Sin, \\
& Torla, Valle de Hecho, Villanova, Yésero. \\
\hline
\end{tabular}

A partir de 1971 fue cuando la oferta se incrementó de forma espectacular, pasando de 5.665 plazas de alojamiento en 1971 a 77.240 plazas en 2008. La Figura 1 muestra las diferencias en la evolución entre los municipios incluidos en el Grupo I y II. Así, en 1971 el Grupo I reunía el 59,9\% del alojamiento, mientras que en 2008 ofertaba el 76\% de las plazas. Por otro lado, se observa que el Grupo II mantiene un crecimiento lineal, mientras que el Grupo I registró un salto muy fuerte en la década de los ochenta (5.188 plazas en 1981 y 34.873 plazas en 1991), para moderar su crecimiento en la década siguiente y retomar un fuerte ritmo durante los últimos años (38.115 plazas en 2001 y 56.690 plazas en 2008).

\subsection{Los efectos de las estaciones de esquí en la población}

En 1950 la población del área de estudio se elevaba a 25.034 habitantes, que se habían reducido a 16.273 habitantes en 1970 y a 15.534 habitantes en 2008. En las últimas seis décadas la población de los Valles del Pirineo Aragonés ha seguido, pues, un claro proceso regresivo. Ahora bien, la Figura 2 pone de manifiesto que los municipios más afectados por las estaciones de esquí muestran durante las primeras décadas una tendencia claramente descendente, que se interrumpe en 1981 cuando inician una evolución ascendente; en la primera fecha alcanzaron su suelo demográfico con 5.141 habitantes, mientras que en 2008 su población ascendía a 8.249 habitantes. Los municipios del Grupo II, por el contrario, registraron su valor más bajo en 1991 (6.756 habitantes), para a partir de esa fecha estabilizar inicialmente su población (6.888 habitantes en 2001) e iniciar un ligero ascenso en los últimos años, hasta alcanzar 7.285 habitantes en 2008, un contingente de sólo el 46,9\% de la población del área de estudio, mientras que en 1950 era del 65,9\%.

La Figura 3 refleja, mediante un gráfico de cajas, la evolución de la población entre 1970 y 2008 en los dos grupos de municipios. Se observa que el rango de la variable es 


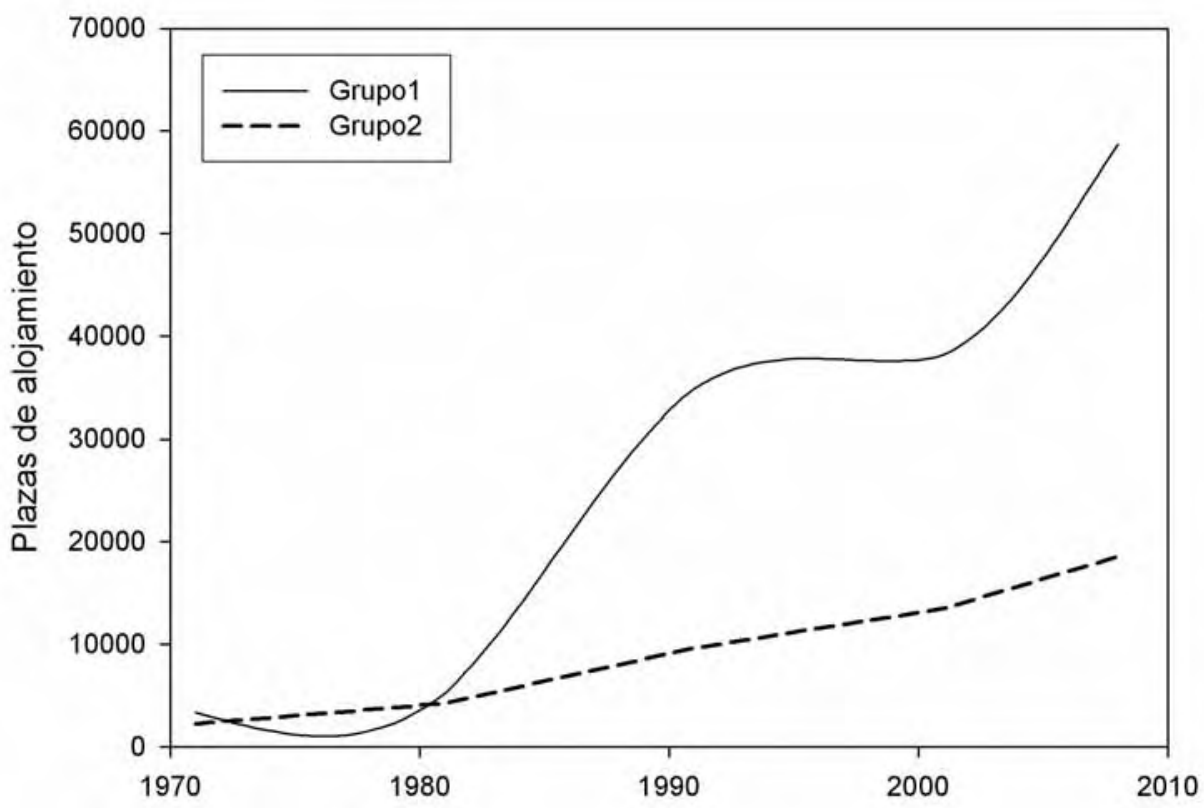

Figura 1. Evolución del alojamiento turístico (1971-2008).

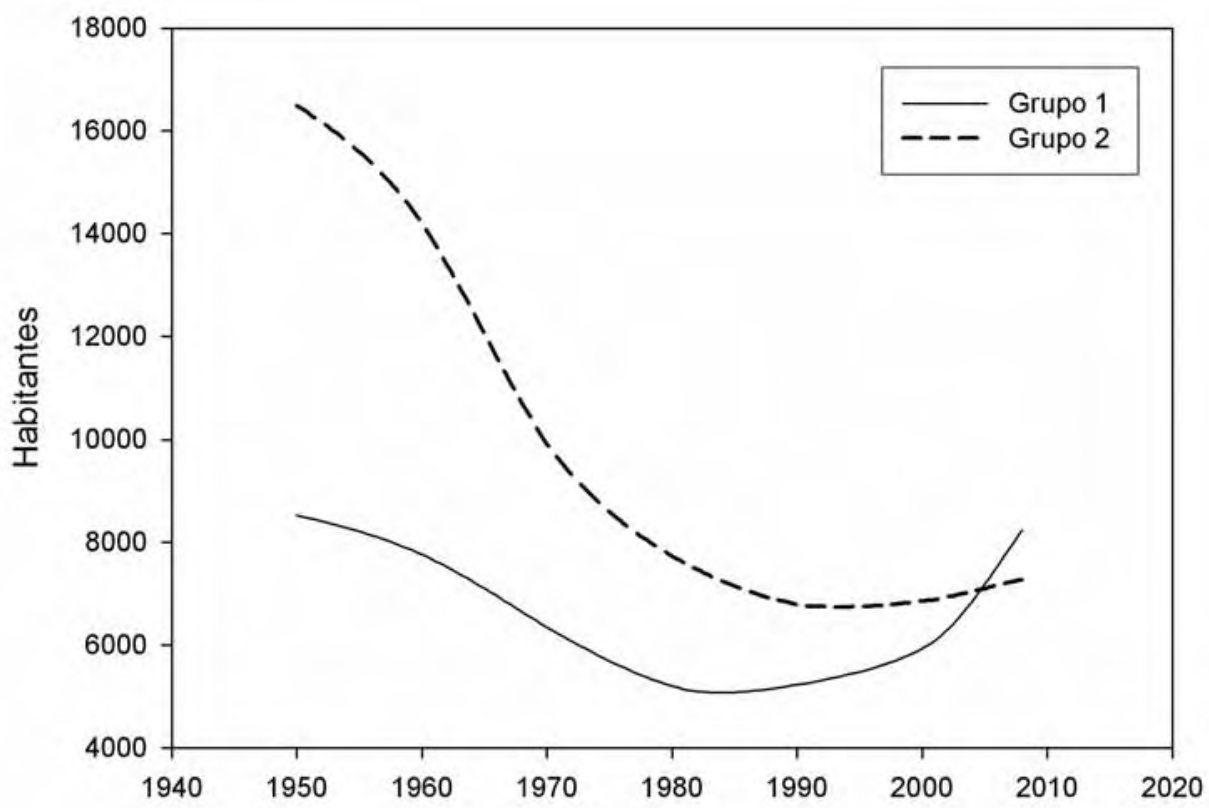

Figura 2. Evolución de la población (1950-2008). 


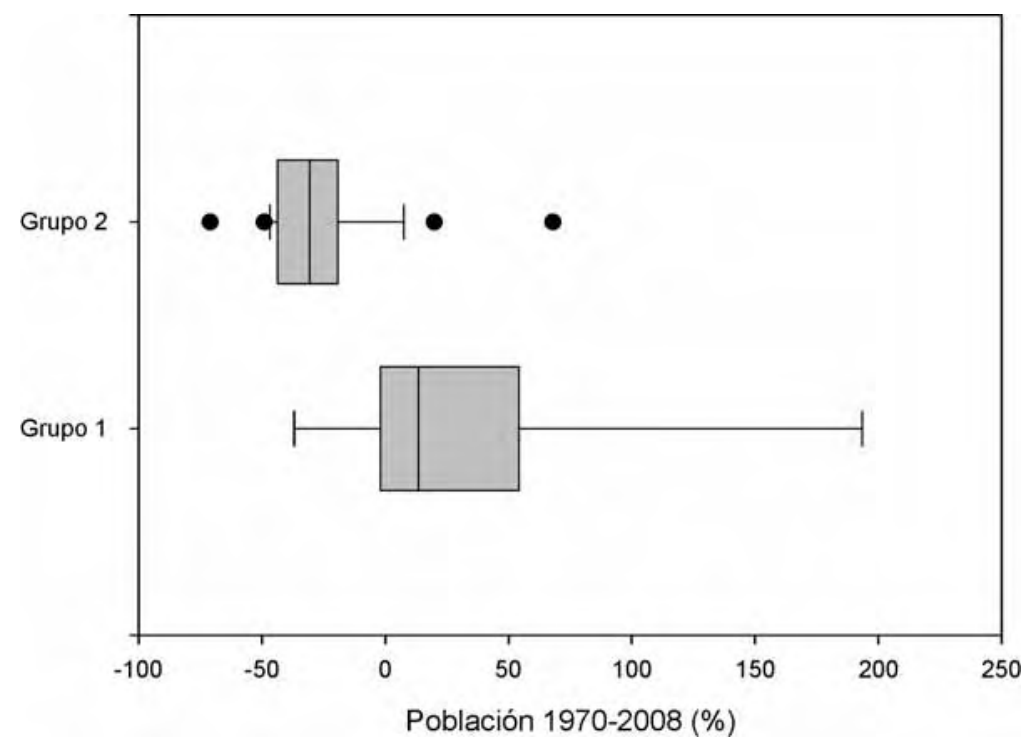

Figura 3. Diagrama de cajas sobre la evolución de la población (1970-2008).

Las barras indican los centiles 10 y 90. Los extremos de la caja los centiles 25 y 75. La línea interior de la caja es la mediana.

superior en el Grupo I que en el Grupo II, debido a que en el primer grupo hay municipios que aumentan su población de forma muy considerable (Benasque 738 habitantes en 1970 y 2.166 habitantes en 2008; Panticosa, 537 y 837, respectivamente), mientras que otros muestran un ligero descenso (Sahún, 372 habitantes en 1970 y 337 en 2008) . En el Grupo II, la varianza es mucho menor, aunque también hay municipios que aumentan su población y otros que pierden habitantes. Lo más interesante, sin embargo, es comprobar que el número de habitantes en el Grupo I aumenta como media el 14\% y que la mayoría de los municipios tienen un comportamiento positivo. Por el contrario, el Grupo II presenta una pérdida media del $32 \%$ en el número de habitantes y todos los municipios, menos dos, dibujan una tendencia demográfica regresiva.

La tabla 3 pone de manifiesto que el grado de envejecimiento es muy superior en el Grupo II que en el Grupo I; en el primer caso el número de personas mayores es de 1.645 (22,6\% de la población total), mientras que en el Grupo I es de 880 personas $(10,7 \%)$. Un análisis más detallado muestra que todos los municipios (menos Aísa) afectados por las estaciones de esquí cuentan con menos del $20 \%$ de personas mayores, mientras que la mayoría de los no afectados supera valores del $20 \%$ e incluso del $30 \%$, alcanzando en Chía el porcentaje del 40,7. Por otro lado, la edad media en el Grupo I es de 42 años, mientras que en el Grupo II es de 49 años. La Tasa de Dependencia muestra también el mejor equilibrio poblacional en el Grupo I, que aporta un valor de 38,5 frente al 55,7 del Grupo II. La Tasa de Feminidad es igual para ambos grupos, no observándose tampoco diferencias a escala municipal. El número de extranjeros del Grupo I es de 1.037 (12,6\% de la población), triplicando casi al del Grupo II (5,6\%). 
Tabla 3. Indicadores de la estructura demográfica en los municipios del área de estudio (año 2008).

\begin{tabular}{|c|c|c|c|c|c|}
\hline Municipio & $\begin{array}{c}\text { \% de población } \\
\text { de }+64 \text { años }\end{array}$ & $\begin{array}{l}\text { Edad } \\
\text { media }\end{array}$ & $\begin{array}{c}\text { Tasa de } \\
\text { Dependencia }\end{array}$ & $\begin{array}{c}\text { Tasa de } \\
\text { Feminidad } \\
\end{array}$ & $\begin{array}{c}\% \text { de } \\
\text { extranjeros }\end{array}$ \\
\hline Aísa & 21,1 & 46,3 & 43,6 & 76,3 & 3,2 \\
\hline Benasque & 8,6 & 37,1 & 26,7 & 88,7 & 13,9 \\
\hline Biescas & 21 & 43,7 & 48,4 & 80,2 & 19,8 \\
\hline Canfranc & 13,1 & 41,2 & 32 & 78,5 & 9,6 \\
\hline Castiello & 19,5 & 44,1 & 45,2 & 82,3 & 5,4 \\
\hline Panticosa & 14,3 & 40,7 & 37,7 & 96,9 & 10,3 \\
\hline Sahún & 19,6 & 43,4 & 45,3 & 91,8 & 15,1 \\
\hline Sallent & 12,7 & 40,2 & 34,8 & 100,3 & 9,8 \\
\hline Villanúa & 14,6 & 42 & 32,7 & 71,7 & 16,5 \\
\hline GRUPO I & 10,7 & 42 & 38,5 & 85,2 & 12,6 \\
\hline Ansó & 32,3 & 50,6 & 68,7 & 101,6 & 2 \\
\hline Aragüés & 28,9 & 50,1 & 54,2 & 88,2 & 2,3 \\
\hline Bielsa & 23,9 & 45,6 & 56,2 & 100 & 13,8 \\
\hline Bisaurri & 26,9 & 50,3 & 50 & 91,8 & 1,3 \\
\hline Bonansa & 19,4 & 45 & 45,3 & 82,4 & 7,5 \\
\hline Borau & 21,9 & 47,9 & 37,7 & 78 & 0 \\
\hline Broto & 20 & 44 & 47,5 & 90,3 & 6,5 \\
\hline Castejón & 16,3 & 40,3 & 48,3 & 87,6 & 15,3 \\
\hline Chía & 40,7 & 54,9 & 88,3 & 85,2 & 4,4 \\
\hline Fago & 32,3 & 51,9 & 72,2 & 82,4 & 3,2 \\
\hline Fanlo & 24,5 & 52,4 & 33,6 & 69,1 & 0 \\
\hline Gistaín & 38,9 & 56 & 78,4 & 84,7 & 0,6 \\
\hline $\mathrm{Hoz}$ & 33,3 & 52,8 & 71,4 & 100 & 2,8 \\
\hline Jasa & 24,6 & 48,4 & 47 & 79,4 & 5,7 \\
\hline Laspaúles & 26,9 & 48,1 & 57,7 & 70,7 & 9,7 \\
\hline Laspuña & 28,1 & 50,3 & 51,8 & 81,9 & 5,6 \\
\hline Montanuy & 27 & 50,6 & 45,5 & 73,4 & 3,9 \\
\hline Plan & 27,7 & 48,5 & 63,7 & 90,2 & 2,1 \\
\hline Puértolas & 22,2 & 46,4 & 40,8 & 83,9 & 9,1 \\
\hline San Juan & 30,7 & 48,1 & 70 & 82,1 & 1,3 \\
\hline Seira & 25,3 & 48,3 & 47,5 & 95,5 & 5,2 \\
\hline Sesué & 21 & 45 & 45,1 & 67,6 & 0,8 \\
\hline Tella-Sin & 33,9 & 52,7 & 66,8 & 81,7 & 10,4 \\
\hline Torla & 24,5 & 48,7 & 48,2 & 93,4 & 1,2 \\
\hline Hecho & 28 & 47,9 & 62,5 & 90,6 & 2,1 \\
\hline Villanúa & 17,8 & 40,8 & 52,4 & 103,9 & 7 \\
\hline Yésero & 35,1 & 56,3 & 54 & 63,8 & 1,3 \\
\hline GRUPO II & 22,6 & 49 & 55,7 & 85,2 & 5,6 \\
\hline
\end{tabular}




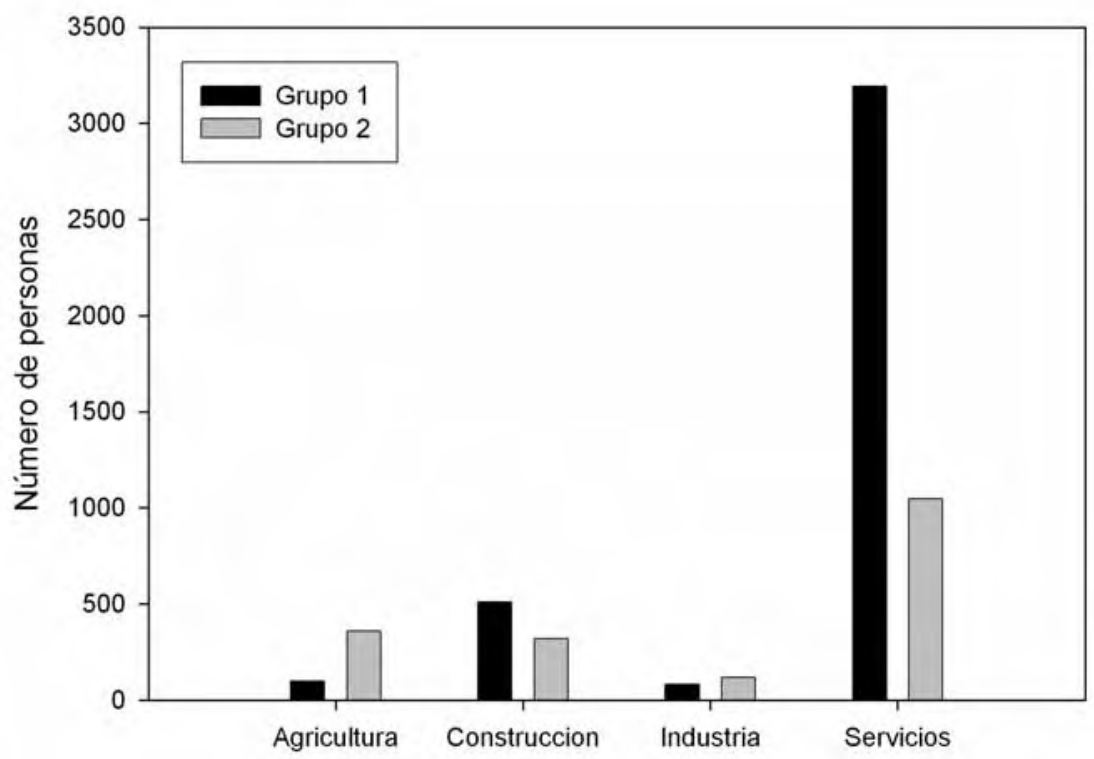

Figura 4. Distribución de la población activa en 2008.

La Figura 4 hace referencia a la distribución de la población activa en ambos grupos de municipios. Llama inicialmente la atención que el número de activos del Grupo I (3.883) duplica al del Grupo II (1.846), como consecuencia de que en el primer caso el 47,1\% de la población total trabaja, mientras que en el segundo sólo lo hace el 25,3\%. La distribución de los activos por sectores muestra un fuerte desequilibrio en el Grupo I, ya que el $82,2 \%$ de los trabajadores se emplean en el sector servicios y el $13,1 \%$ en la construcción, muy ligada al turismo (edificación de segundas residencias, hoteles, complejos deportivos...), mientras que en el primario sólo trabaja el 2,6\% de los activos y en la industria el 2,1\%. En el Grupo II hay un mayor equilibrio entre sectores económicos, debido al mantenimiento del sector primario (19,5\% de los activos) y una tímida presencia de la industria, que ocupa al 6,5\% de los trabajadores. Aun con todo, el sector servicios domina claramente $(56,7 \%)$, seguido por la construcción $(17,3 \%)$.

\subsection{Los efectos de las estaciones de esquí en las actividades tradicionales}

Los cambios en el sector primario se han analizado mediante gráficos de cajas. La Figura 5 relaciona la evolución del número de explotaciones entre 1970 y 2008. Se observa que ambos grupos pierden explotaciones, mostrando un comportamiento acorde con la tendencia general seguida por el mundo rural español. No obstante, existen diferencias interesantes; así, el Grupo I pierde el $48 \%$ de sus explotaciones, mientras que el Grupo II sólo pierde el 22\%. Por otro lado, todos los municipios del Grupo I han visto reducido su censo de explotaciones, mientras que en el Grupo II hay municipios que pierden y otros que ganan explotaciones. 


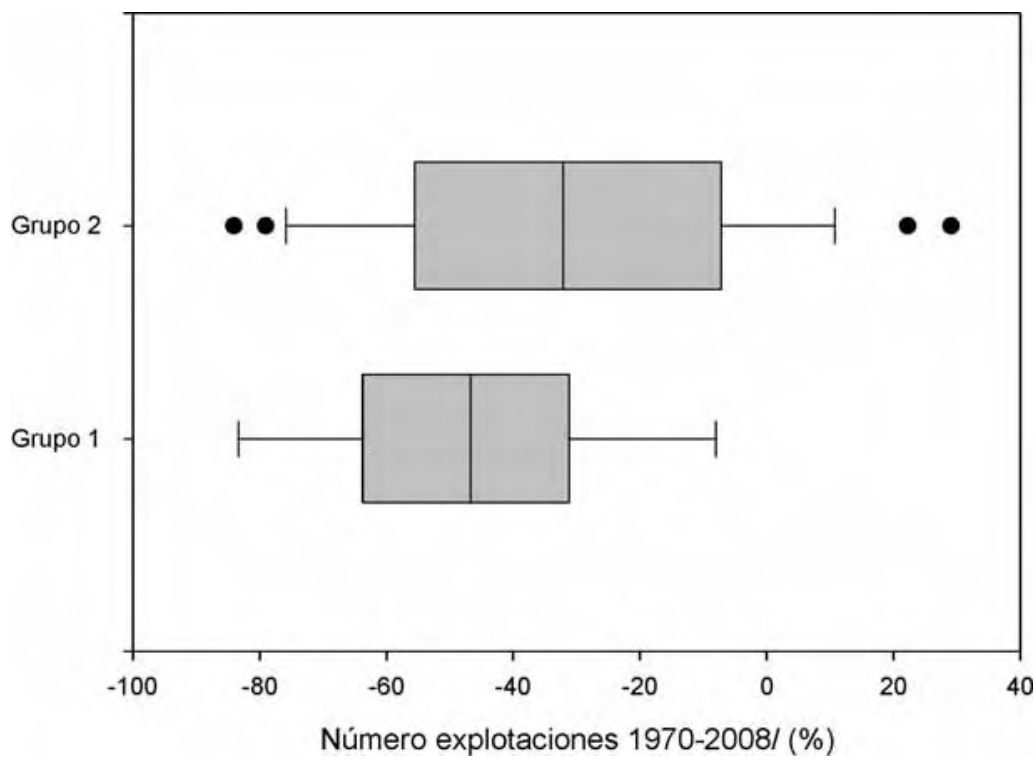

Figura 5. Diagrama de cajas sobre la evolución del número de explotaciones (1970-2008). Las barras indican los centiles 10 y 90. Los extremos de la caja los centiles 25 y 75. La línea interior de la caja es la mediana.

La Figura 6 hace referencia a la evolución del censo ganadero. El Grupo I presenta pérdidas medias del $43 \%$ en el número de cabezas, pasando de 9.789 UGM en 1970 a 5.605 UGM en 2008, con descensos muy acusados, por ejemplo, en Biescas (2.307 UGM en 1970 y 1.227 UGM en 2008), Panticosa (1.109 y 637 UGM, respectivamente) y Sallent (3.331 y 1.038 UGM, respectivamente). El Grupo II, por el contrario, ha incrementado su censo en el 31,8\%, pasando de 18.116 UGM en 1970 a 26.545 UGM en 2008, con incrementos notables en algunos municipios ribagorzanos: Bisaurri (1.112 UGM en 1970 y 3.749 UGM en 2008), Chía (250 y 517 UGM, respectivamente), Laspaúles (1.494 y 1.718 UGM, respectivamente), Montanuy (2.356 y 4.503 UGM, respectivamente), Valle de Plan (1.598 y 2.421 UGM, respectivamente), Broto (1.048 y 1.876 UGM, respectivamente), Hecho (1.952 y 2.631 UGM, respectivamente) y Puértolas (409 y 1.639 UGM, respectivamente). La Figura 6 también pone de relieve que en el Grupo I la mayoría de los municipios registran pérdida de efectivos ganaderos, pues la caja del gráfico presenta signo negativo, mientras que en el Grupo II ocurre lo contrario, con una caja de signo claramente positivo, debido a que muy pocos municipios sufrieron pérdidas.

Las estaciones de esquí, con el consiguiente incremento de la actividad turística, no contribuyen, pues, a incrementar ni a mantener los censos ganaderos y las explotaciones primarias. El retroceso de la ganadería en relación con el crecimiento turístico debe atribuirse a la competencia que se establece entre ambas actividades por el uso de la mano de obra y de los espacios más fértiles, dedicados al cultivo de prados. Ya se ha señalado que en los municipios con estaciones de esquí sólo el 2,6\% de la población trabaja en el sector 


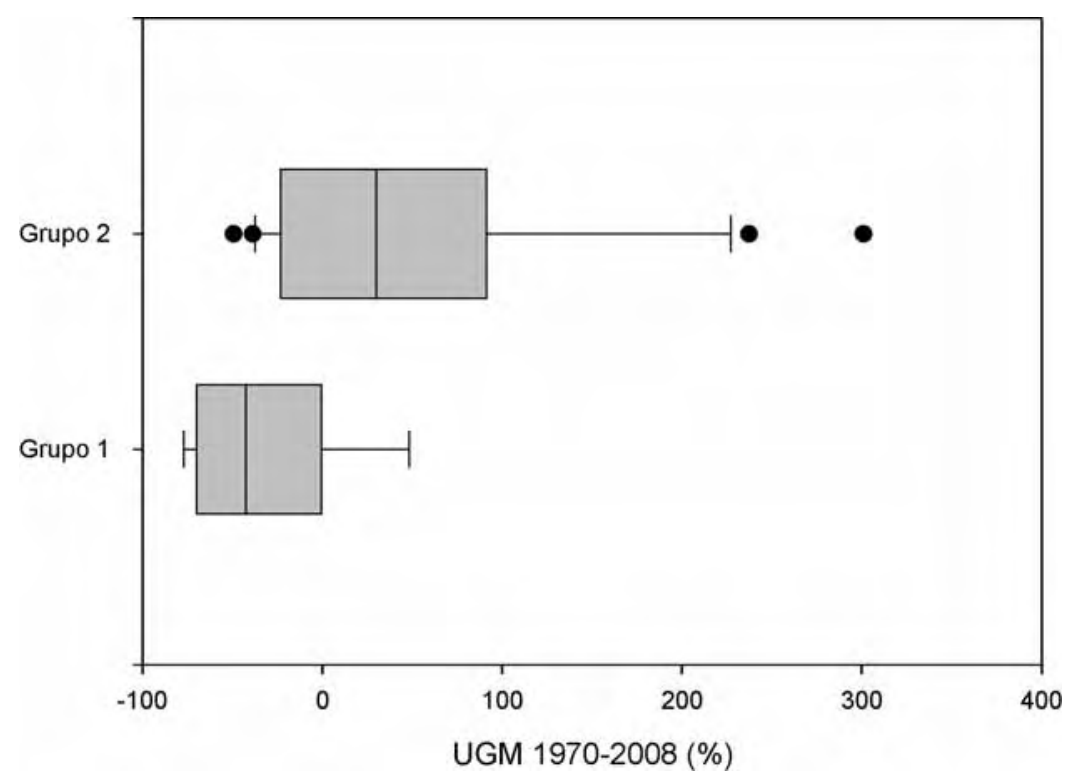

Figura 6. Diagrama de cajas sobre la evolución del censo ganadero (1970-2008). Las barras indican los centiles 10 y 90. Los extremos de la caja los centiles 25 y 75 . La línea interior de la caja es la mediana.

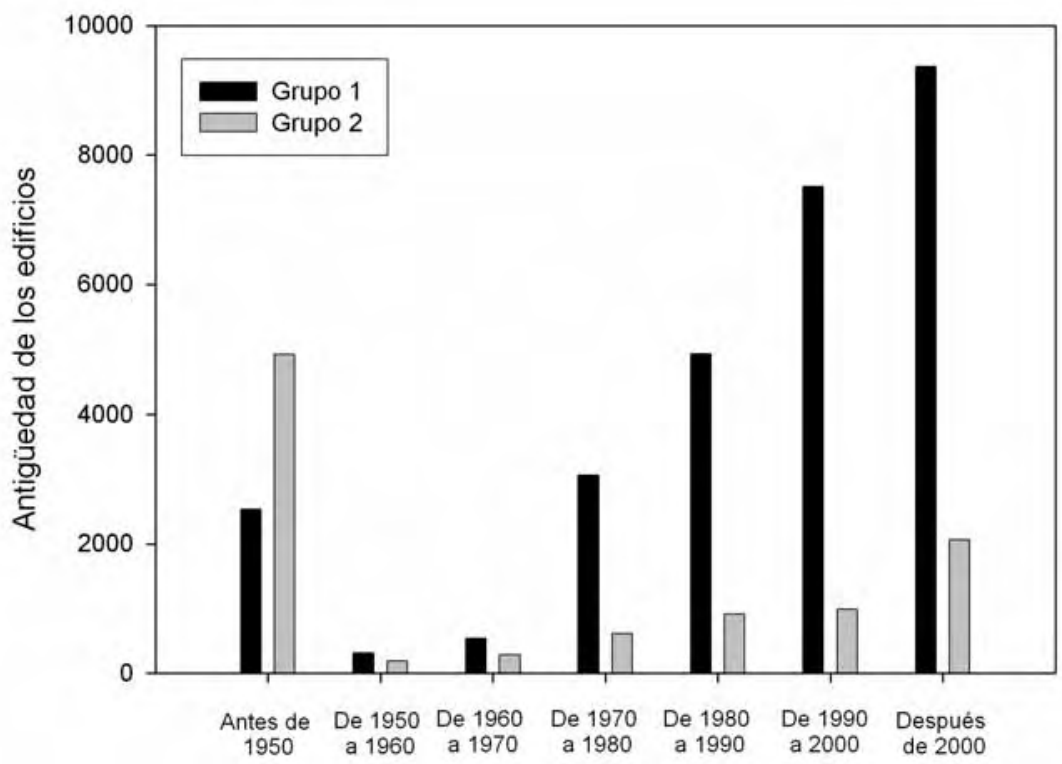

Figura 7. Edad de edificación de las viviendas. 
primario, mientras que lo hace el 19,5\% en los no afectados por las estaciones de esquí. El tema fue estudiado con detalle en el Alto Esera, donde se localiza la estación de Cerler. Laguna y Lasanta (2003) comprobaron que en Benasque y Sahún había mayor trasvase de población desde el sector primario al terciario que en el resto de los municipios del Valle. Así, Benasque mantenía en 2000 sólo el 16\% de las explotaciones de 1965, mientras que Sesué (con apenas actividad turística) mantenía el 46\% de las explotaciones. En el mismo sentido, en Benasque el 37\% de los empleados en el sector primario en 1965 trabajaba en 2000 en actividades turísticas, mientras que en Sesué sólo lo hacía el 16\%.

Por otro lado, entre el sector primario y terciario se establece la competencia por los espacios localizados en los fondos de valle y próximos a los pueblos y vías de comunicación. Con frecuencia, tales espacios constituyen las áreas más fértiles por ser campos llanos, cultivados durante siglos y receptores de abonado frecuente (Lasanta, 1989). Sin embargo, también son los más apetecibles para el sector turístico, al ser los más próximos a los servicios necesarios para su urbanización (red de saneamiento, electricidad, accesos,...), por lo que con frecuencia son ocupados por infraestructuras turísticas (urbanizaciones, campings, complejos deportivos, vías de comunicación...), lo que resta un espacio productivo que suele ser determinante para la viabilidad económica de no pocas explotaciones agroganaderas. La sustracción de algunos campos implica la venta de cabezas de ganado, con el fin de equilibrar en las explotaciones el censo y la producción propia de pienso para garantizar la alimentación durante la estación fría; así cada explotación cuenta como media con 15,4 UGM en los municipios del Grupo I por 29,6 UGM en el Grupo II. El pequeño tamaño de las explotaciones en los municipios turísticos plantea con frecuencia problemas de rentabilidad, que conduce a la venta del ganado y al abandono o arrendamiento de los prados, en definitiva al cese de la explotación (Laguna y Lasanta, 2003 a).

La Figura 7 hace referencia a la edad de construcción de las viviendas en los dos grupos de municipios. Antes de 1950 el número de viviendas del Grupo II (4.913) casi duplicaba a las del Grupo I (2.533). Sin embargo, la evolución desde esa fecha ha sido muy diferente, especialmente desde los años setenta, cuando los municipios con estaciones de esquí muestran un crecimiento exponencial, que ha llevado a un censo total de 28.225 viviendas, de las que 9.365 (el 33,2\%) se han construido después del año 2000. Por el contrario, los municipios poco o nada afectados por las estaciones de esquí han crecido de forma más moderada hasta completar un censo de 9.949 viviendas. El diferente ritmo de construcción implica que el suelo urbano ocupa 761,8 ha (367,7 ya edificadas y 394,1 de solares) en el Grupo I, mientras que se extiende por 336,8 ha (256,1 edificadas y 80,7 de solares) en el Grupo II, superficies que se han extraído fundamentalmente del área de prados.

\section{Discusión y conclusiones}

Desde los años sesenta del siglo XX el Pirineo español trata de potenciar su desarrollo impulsando la actividad turística, después de que el sistema tradicional de gestión del territorio, basado en la ganadería extensiva, entrase en una profunda crisis (García- 
Ruiz y Lasanta, 1990). La estrategia emprendida considera a las estaciones de esquí como el principal motor de desarrollo, siguiendo el modelo de las montañas centroeuropeas (Barrère, 1981; Messerli, 1987; Godde et al., 2000).

Transcurridos más de 30 años desde la inauguración de las últimas estaciones de esquí, resulta interesante conocer el efecto que han tenido en el desarrollo socioeconómico de sus áreas de influencia. En este trabajo se ha comprobado que en el Pirineo Aragonés los resultados son positivos respecto a la situación demográfica y negativos en la evolución del sector primario. Se observa que los municipios con estaciones de esquí incrementan su población, pasando de 5.141 habitantes en 1981 a 8.249 habitantes en 2008. El incremento de población se debe a la llegada de inmigrantes procedentes de otros municipios de la misma región (Daumas, 1986), de neorrurales que han montado un negocio ligado al turismo (Loscertales, 1993), y de extranjeros: en 2008 el 12,6\% de la población censada en el Grupo I había nacido fuera de España. Los inmigrantes son población en edad de trabajar y frecuentemente con hijos, lo que explica que los municipios más afectados por el turismo tengan una estructura demográfica más equilibrada: menor porcentaje de personas mayores, menor tasa de dependencia y más baja edad media de la población. El Pirineo aragonés sigue en este aspecto la misma tendencia que otras montañas con elevada presencia de la industria turística (Buckely et al., 2000; Pechlaner and Tschurtschenthaler, 2003).

Por el contrario, los municipios sin estaciones de esquí siguen inmersos en la tendencia demográfica negativa iniciada décadas antes, con la pérdida progresiva de población y desestructuración demográfica. La distinta evolución demográfica muestra un primer contraste espacial entre municipios, diferenciando entre los que han detenido la sangría demográfica y los que siguen inmersos en el proceso de despoblación. Este contraste tiene destacadas consecuencias de cara al desarrollo, ya que los municipios con bajo número de habitantes y pocos jóvenes necesitan gran número de servicios sociales y apenas tienen posibilidades de progresar económicamente.

La evolución de la población activa refleja también la disarmonía entre los municipios "beneficiados" por las estaciones de esquí y los no afectados. El número de trabajadores en el sector terciario es muy elevado en los primeros $(82,2 \%$ de la población activa), que apenas conservan activos en el primario (2,6\%). Por el contrario, los municipios no afectados por las estaciones de esquí presentan una distribución más equilibrada entre sectores, manteniendo mejor el sector primario (19,5\% de los activos).

El contraste en la distribución de la población activa se explica porque los inmigrantes vienen para trabajar en el sector servicios o en trabajos muy vinculados (construcción fundamentalmente). Laguna y Lasanta (2003 b) señalan que en Benasque (municipio donde se localiza la estación de esquí de Cerler) había 42 explotaciones primarias en 1965. En el año 2000, el 14\% de sus propietarios o descendientes mantenía la explotación, el $41 \%$ trabajaba en actividades ligadas al turismo, el 21\% había emigrado y el $24 \%$ de las explotaciones había desaparecido por falta de continuidad. Lluelles (1991) llega a la conclusión de que en Andorra se ha producido una concentración de la población activa en torno al turismo de nieve, lo que ha acarreado la casi desaparición 
de los activos primarios. Este hecho también se comprobó en los Alpes franceses, donde Preau (1984) contabilizó que tan sólo el 6,3\% de los jóvenes se mantenían en el primario, mientras que el $91 \%$ pasaban a trabajar en actividades ligadas con el turismo.

La evolución en el sector primario muestra también claras diferencias entre los dos grupos de municipios. Todos han perdido explotaciones siguiendo la tendencia general del mundo rural español, que desde mediados del siglo XX sufre una pérdida muy acusada de explotaciones primarias en el tránsito de una sociedad rural a otra industrial (Pérez Yruela, 1995). Sin embargo, los vinculados a las estaciones de esquí han perdido mayor proporción de explotaciones que los no vinculados, porque ha habido un trasvase de trabajadores del sector primario a la actividad turística.

Más claros son los contrastes en la evolución de los censos ganaderos. El área de estudio muestra un ligero incremento, pasando de 27.905 UGM en 1970 a 32.150 UGM en 2008. Ello se debe fundamentalmente a los incentivos de la Política Agraria Comunitaria, que a través de las ayudas económicas han favorecido el aumento del tamaño de las explotaciones (Laguna, 2006). Sin embargo, el crecimiento no ha sido homogéneo espacialmente, centrándose en los municipios sin vinculación con las estaciones de esquí, que han incrementado su censo en el 31,8\%. Hay que destacar que algunos de los valles más alejados de las estaciones de esquí (Noguera Ribagorzana, Hecho, Plan, Puértolas y Broto) son los que registraron los mayores incrementos en los censos ganaderos. Por el contrario, los municipios afectados por las estaciones de esquí registraron una disminución del $43 \%$ en su cabaña. Laguna y Lasanta (2003 a) señalan que en los municipios con fuerte crecimiento turístico, además de haber un trasvase de mano de obra del primario al terciario, se produce la ocupación de prados por infraestructuras turísticas perdiéndose unos campos que con frecuencia son determinantes para la viabilidad económica de algunas explotaciones. Hay que tener en cuenta que tras la crisis del sistema trashumante el espacio clave para el mantenimiento de la ganadería son los prados, habiendo una relación muy directa entre superficie de prados y censos ganaderos (García-Ruiz y Lasanta, 1993). Se confirma, pues, en el Pirineo lo que es un hecho ya relativamente conocido en otras montañas europeas: la competencia entre el turismo y el sector primario por el uso de las áreas más fértiles y por la mano de obra (Oberacher, 1995; Snowdon et al., 2000).

La competencia entre el turismo y las actividades primarias plantea limitaciones para un desarrollo sostenible, que debe basarse en la complementariedad de ambas. Si las actividades turísticas terminan por ahogar al sector primario se desaprovecharán y perderán los pastos naturales, un recurso todavía abundante y barato que permite utilizar y rentabilizar el territorio con ganadería extensiva (Bernués et al., 2005). Además, el monocultivo turístico conduciría a la degradación del paisaje (un activo destacado de la economía pirenaica), creado y moldeado por el hombre durante siglos de utilización agropecuaria (Galop, 1998). La disminución drástica de la ganadería extensiva conduce a la subutilización de pastos naturales y al abandono de prados, lo que permite el avance de matorrales y bosques de sucesión y conduce a la disminución de la diversidad paisajística (Vicente-Serrano et al., 2000), con consecuencias en el incremento del riesgo de incen- 
dios, en la conservación del suelo, en la disponibilidad de agua en las cuencas y en el atractivo turístico (Buckley et al., 2000; Lasanta et al, 2005; García-Ruiz et al., 2008).

El problema de la marginación socioeconómica y abandono de la montaña no se resuelve simplemente con facilitar el trasvase de la mano de obra agropecuaria al sector terciario, sino que además hay que hacer lo posible por mantener una productividad primaria sostenida. En este sentido, parece que proyectos de gran envergadura, como las estaciones de esquí del Pirineo aragonés, son poco asimilables por la población local, muy escasa y envejecida, y conducen al cese de explotaciones y a la disminución de los censos ganaderos, con la consiguiente pérdida de recursos naturales y degradación paisajística. Sin embargo, su elevado potencial turístico obliga a buscar fórmulas de desarrollo que compatibilicen el turismo con el aprovechamiento agroganadero del territorio, el único capaz de generar rentas y de mantener simultáneamente la biodiversidad y el paisaje, requisitos imprescindibles para la sostenibilidad de áreas donde la naturaleza es su principal recurso.

\section{Agradecimientos}

Este trabajo se ha realizado en el marco de los proyectos de investigación: "Procesos y balances de sedimentos a diferentes escalas espaciales en ambientes mediterráneos: Efectos de las fluctuaciones climáticas y de los cambios de uso del suelo" (CGL200611619/HID), y "Análisis de escenarios de riesgo a escala nacional y global” (CGL200801083/CLI), ambos financiados por el Plan Nacional de I+D. También ha contado con la ayuda del "Programa de grupos de investigación consolidados", financiados por el Gobierno de Aragón.

\section{Referencias bibliográficas}

Arnáez, J. (1981). Pautas del comportamiento del turismo en la estación de esquí de Valdezcaray (Rioja). Cuadernos de Investigación Geográfica, 7 (1-2): 101-114.

Balcells, E. (1976). El Pirineo: contraste de paisajes; enlace de pueblos. Comisión Internacional de los Pirineos. Imprenta del Ministerio de Asuntos Exteriores, 77 pp., Madrid.

BARRÈre, P. (1981). La haute montagne, bien de nature où objet de profit. En. Supervivencia de la montaña. Ministerio de Agricultura, pp. 459-475, Madrid.

Bernués, A., Riedel, J. L., Asensio, M. A., Blanco, M., Sanz, A. Revilla, R., Casasús, I. (2005). An integrated approach to studying the role of grazing livestock systems in the conservation of rangelands in a protected natural park (Sierra de Guara, Spain). Livestock Production Science, 96: 75-85.

Buckley, R. C., Pickering, C. M., Warnken, J., Goode, P. M. (2000). Environmental management for alpine tourism and resorts in Australia. In: Tourism and development in mountain regions (Price, M.F., Zimmermann, F.M., Eds.), pp. 27-45, Wallingford 
Callizo, J. (1991). Aproximación a la Geografía del Turismo. Síntesis: 215 pp., Madrid.

Caravaca, I., Méndez, R. (1994). Industrial revitalization of the metropolitan areas in Spain. International Journal of Urban and Regional Research, 18: 220-232.

DAumas, M. (1986). La redistribution géographique de la population dans les hautes vallées montagnardes: l'exemple du val de Benasque. Revue de Géographie Alpine, 64: 189-196.

Del Barrio, G., Creus, J., Puigdefábregas, J. (1990). Termal seasonality of high mountain belts on the Pyrenees. Mountain Research and Development, 10, 227-233.

Domingo, S. (2000). La nieve en Aragón. CAI 100, pp. 110, Zaragoza.

FERrero, G. (1998). Seconde case, politiche urbanistiche e turismo nelle Alpi occidentali italiane. Revue Géographie Alpine, 86: 61-68.

Galop, D. (1998). La forêt, l'homme et le troupeau dans les Pyrénées. 6000 ans d'histoire de l'environment entre Garonne et Méditerranée. GEODE - Laboratoire d'Ecologie Terrestre-Franespa, pp. 285, Toulouse.

García-Ruiz, J. M., Balcells, E. (1978). Tendencias actuales en la ganadería del Alto Aragón. Estudios Geográficos, 153: 539-560.

García-Ruiz, J. M., Lasanta, T. (1990). Land-use changes in the Spanish Pyrenees. Mountain Research and Development, 10: 267-279.

García-Ruiz, J. M., LASANTA, T. (1993). Land use conflicts as a result of land use change in the Central Spanish Pyrenees: a review. Mountain Research and Development, 13: 295-304.

García-Ruiz, J. M., SAla, M. (1984). Pyrenees and Ebro Basin Complex. En: Gemorphology of Europe. Macmillan Reference Books, pp. 495, London.

García-Ruiz, J. M., Puigdefábregas, J., Creus, J. (1985). Los recursos hídricos superficiales del Alto Aragón. Instituto de Estudios Altoaragoneses. Colección de Estudios Altoaragoneses, pp. 224, Huesca.

García-Ruiz, J. M., Puigdefábregas, J., Creus, J. (1986): La acumulación de la nieve en el Pirineo Central y su influencia hidráulica. Pirineos, 127: 27-72.

García-Ruiz, J. M., Regüés, D., Alvera, B., Lana-Renault, N., Serrano-Muela, P., Nadal-Romero, E., Navas, A., Latrón, J., Martí-Bono, C., Arnáez, J. (2008). Flood generation and sediment transport in experimental catchments affected by land use changes in the Central Pyrenees. Journal of Hydrology, 356: 245-260.

Godde, P. M., Price, M. F., Zimermman, F. M. (2000). Tourism and development in mountain regions. Oxon, CABI Publishing.

Laguna, M. (2006). Las políticas de desarrollo rural en el Pirineo Aragonés. Ediciones Trea, pp. 239, Gijón. 
Laguna, M., Lasanta, T. (2001). El papel de la Administración en la ampliación espacial de la oferta turística: el caso del Pirineo Aragonés. X Coloquio de Geografía Rural. Asociación de Geógrafos Españoles, 422-447 pp., Lérida.

Laguna, M., Lasanta, T. (2003 a). Competing for meadows. A case study on tourism and livestock farming in the Spanish Pyrenees. Mountain Research and Development, 23: 169-176.

Laguna, M., Lasanta, T. (2003 b). Influencia del desarrollo turístico en la ganadería pirenaica: competencia por el uso de la mano de obra y por el espacio agrícola. Estudios Geográficos, 250: 23-45.

Lasanta, T. (1989). Evolución reciente de la agricultura de montaña: el Pirineo aragonés. Geoforma Ediciones, pp. 220, Logroño.

Lasanta, T., Laguna, M. (2002). Desarrollo turístico y sostenibilidad en el Pirineo aragonés: efectos opuestos del modelo dominante. En: Los espacios rurales entre el hoy y el mañana. XI Coloquio de Geografía Rural. AGE, 149-157 pp., Santander.

Lasanta, T., Laguna, M., Vicente-Serrano, S. (2007). Variabilidad espacial de los efectos socioeconómicos de las estaciones de esquí alpino en los municipios rurales del Pirineo aragonés. Pirineos, 162: 155-176.

Lasanta, T., Vicente-Serrano, S., Cuadrat, J. M. (2005). Mountain mediterranean landscape evolution caused by the abandonment of traditional primary activities: a study of the Spanish Central Pyrenees. Applied Geography, 25: 47-65.

LóPEZ-Moreno, J. I., GARCíA-RuIZ, J. M. (2004). Influence of snow accumulation and snowmelt on streamflow in the Central Spanish Pyrenees. Hydrological Sciences Journal, 49: 787-802.

López-Moreno, J. I., García-Ruiz, J. M., Beniston, M. (2008). Environmental change and water management in the Pyrenees. Facts and future perspectives for Mediterranean mountains. Global and Planetary Change, 66(3-4): 300-312.

LóPeZ-Moreno, J. I., Vicente-SERrano, S., LANJeri, S. (2007). Mapping of snowpack distribution over large areas using GIS and interpolation techniques. Climate Research, 33: $257-270$.

Loscertales, B. (1993). Jacetania. De espacio agrario a espacio turístico. Prames: pp. 319, Zaragoza.

Lluelles, M. J. (1991). La transformació económica d'Andorra. Coleccio Clio-11: 690 pp., Barcelona.

Messerli, P. (1987). The development of tourism in the Swiss Alps: economic, social and environmental effects. Experience and recommendations from the Swiss Mab programme. Mountain Research and Development, 7: 13-24.

Oberacher, A. (1995). Agriculture and tourism in the Alps. Conflict or symbiosis. Forderungsdient, 43: 109-115. 
Pechlaner, H., Tschurtschenthaler, P. (2003). Tourism policy, tourism organizations and change management in Alpine regions and destinations: A European Perspective. Current Issues in Tourism, 6: 508-539.

Pérez Yruela, M. (1995). Spanish rural society in transition. Sociologia Ruralis, 35: 276-296.

Preau, P. (1984). Le changement social dans une commune touristique de montagne: Saint-Bon-Tarentaise (Savoie). Deuxième article. Revue Géographie Alpine, 72: 411-437.

Snowdon, P., Slee, B., Farr, H., Godde, P. M. (2000). The economic impacts of different types of tourism in upland and mountain areas of Europa. In: Tourism and Development in Mountain Regions (Godde, P.M., Price, M.P., Zimmermann, F.M., Eds.). Wallingford: CAB International, 137-145 pp.

Vicente-Serrano, S., Lasanta, T., Cuadrat, J. M. (2000). Transformaciones en el paisaje del Pirineo como consecuencia del abandono de las actividades económicas tradicionales. Pirineos, 155: 111-133.

Zimmermann, F. (1994). El papel del turismo en la reestructuración de las regiones alpinas. Papers de Turismo, 16: 77-87. 